in print (2016)

(- Already online -)

Marco Brigaglia

\title{
Rules and norms
}

Two kinds of normative behaviour

\begin{abstract}
Warning
The contents of this site is subject to the French law on intellectual property and is the exclusive property of the publisher.

The works on this site can be accessed and reproduced on paper or digital media, provided that they are strictly used for personal, scientific or educational purposes excluding any commercial exploitation. Reproduction must necessarily mention the editor, the journal name, the author and the document reference.

Any other reproduction is strictly forbidden without permission of the publisher, except in cases provided by legislation in force in France.
\end{abstract}

\section{revues.org}

Revues.org is a platform for journals in the humanities and social sciences run by the CLEO, Centre for open electronic publishing (CNRS, EHESS, UP, UAPV).

\section{Electronic reference}

Marco Brigaglia, « Rules and norms », Revus [Online], in print | 2016, Online since 23 July 2016, connection on 27 September 2016. URL : http://revus.revues.org/3447 ; DOI : 10.4000/revus.3447

Publisher: Klub Revus - Center za raziskovanje evropske ustavnosti in demokracije http://revus.revues.org

http://www.revues.org

Document available online on:

http://revus.revues.org/3447

Document automatically generated on 27 September 2016. The page numbering does not match that of the print edition.

All rights reserved 


\section{Marco Brigaglia}

\section{Rules and norms}

\section{Two kinds of normative behaviour}

Pages in print edition : $\mathrm{p}$.

\section{Introduction}

There do exist, Celano argues, entities which can be characterised as "normative facts": regularly followed patterns of action (factual regularities) which, as such, constitute standards of correctness (norms). "Pre-conventions" are a peculiar kind of such entities. Following Celano, a pre-convention is a social structure defined by the following conditions.

(1) Members of a social group G (let us call them "the Gs") behave regularly, performing actions that satisfy a certain action type A, in circumstances that satisfy a certain situation type S. Let us say, in short, that the Gs replicate the behavioural scheme "A in S". In this respect, "A in S" is a factual regularity amongst the Gs.

(2) The replication of "A in S" by the Gs is "automatic": it happens without reasoning and deliberate choice, and is, as such, different from and irreducible to the observance of the "rule", or the system of rules, which prescribes the doing of A in S. In doing A in S, therefore, the Gs are not following a rule or a system of rules (at least not in the most familiar sense of "following a rule", see below, § 2.1). In this respect, "A in S" is not (it does not function as) a rule.

(3) "A in S" is, nevertheless, not a simple factual regularity. Indeed, for the Gs, it counts as a standard of correctness: the Gs consider those concrete actions which, in S, satisfy A to be correct, while those which do not to be incorrect; and this very fact contributes causally to them replicating A in S. In doing automatically A in S, the Gs can therefore be said to be conforming to the standard of correctness "A in S", even if they are not following the rule which prescribes the doing of A in S. Relying on a terminological difference emerging from Celano's paper, we can express this point by saying that they are conforming to a "norm". 2 "A in $\mathrm{S}$ " is (it does function as) a norm.

(4) The norm "A in S" is not an innate scheme, ${ }^{3}$ a biological invariant (such as a hungry baby's sucking anything sufficiently similar to a woman's breast). It is indeed - in some sense, and in part at least - a contingent (not necessary, arbitrary) social construction.

(5) Finally, I assume (although Celano is not explicit about this point) that, for the existence of a pre-convention, the "dependence condition"4 should hold: the Gs conform to the norm "A in S" (at least in part) because... the Gs conform to the norm " $\mathrm{A}$ in $\mathrm{S}$ ". (The relation is meant to be a merely causal one: the fact that the Gs conform to the norm "A in S" causes the Gs to conform to the norm "A in $\mathrm{S}$ ".)

Pre-conventions are, thus, normative social structures different from, irreducible to, structures of rules: they are, instead, structures of norms. Celano further argues that pre-conventions have a pervasive presence in social life: they are part of the background, which other kinds of social practices, including the shared recognition and application of a rule or a system of rules, are necessarily built upon. Our pre-conventions are (part of) the background of our rules.

I have nothing to object to Celano's theses and arguments: I find both fully convincing. I shall rather focus on the distinction - presupposed in the above definition of a pre-convention between two different kinds of normative (i.e., normatively guided) behaviour, observing a rule and conforming to a norm, and the underlying different kinds of normative standards, rules and norms.

The point is clearly critical for the purpose of Celano's paper: it is a definitional property of preconventions, distinguishing them from other types of conventions, that they are networks of 
converging normative behaviours which do not consist in "observing rules", either consciously or unconsciously, but rather in "conforming to norms".

Nevertheless, Celano does not develop a comprehensive and detailed account of how the distinction should be understood. Indeed, he provides various intriguing, yet vague and fragmentary, connotations and examples - observing a rule involves "reasoning", while conforming to a norm is an "automatic", "spontaneous", "fluid and effortless" process; rules are "explicit", "intentional", "propositional", while norms are "tacit", "embodied", "not intentional"; paradigmatic examples of conforming to norms are swimming the front crawl, marching, acting with style, ascribing an entity to a concept, etc. - apparently aiming at suggesting rather than minutely analysing the notions. Even the different ways in which the terms "rules" and "norms" are actually used in the paper are not explicitly introduced. It is my impression, however, that a more complex and rich design lies in the background. Celano's suggestions implicitly set rather clear and precise criteria for distinguishing between the two kinds of normative behaviour, observing rules and conforming to norms; moreover, based on this distinction, they trace a general account of rule-following, which seems to me to be, albeit sketchy, highly coherent, plausible, and far from obvious. I shall call it the Rules vs Norms Framework.

My aim in this article is to provide a systematic reconstruction of the Rules vs Norms Framework. I expect it can usefully contribute to the present discussion by helping to better understand, but also to support, Celano's theses, pointing out the solid structure that they rely upon and providing an interpretation of the crucial, yet not completely clear points (as, for instance, the distinction between "explicit" and "tacit" mental states, or the sense in which pre-conventions can be said to be "embodied"). I think, however, that, besides its key role in structuring the arguments put forward in Pre-conventions, the Rules vs Norms Framework deserves, on its own, to be made explicit: it, as such, constitutes one of the paper's main achievements, and marks a decisive step in Celano's intellectual trajectory, pointing at very promising paths for his future work.

In section 2, I shall propose my reconstruction of the Rules vs Norms Framework. In section 3 , I shall focus on its importance and foreseeable (and desirable) applications, and introduce a methodological issue, advancing a (marginal) criticism of Celano's jargon.

\section{The Rules vs. Norms Framework}

The above definition of a pre-convention presupposes the distinction between two kinds of normative behaviour. By "normative behaviour" I mean any action satisfying some standard of correctness and performed under its guidance or control, considered together with and categorised with reference to the mental process leading to its execution. I include in the notion not only external, "bodily" actions, but also "mental" actions, such as the ascription of a meaning to a sentence, the ascription of an entity to a concept, the drawing of a conclusion from an inference, and so on. ${ }^{5}$

Emphasising Celano's use of the terms "rules" and "norms", I shall call the first kind (the absence of which characterises a pre-convention) "rule-guided behaviour" or "explicit" rulefollowing (hereafter, R-behaviour), and the second (the occurrence of which characterises a pre-convention) "norm-conforming behaviour" or "tacit" rule-following (hereafter, Nbehaviour).

\section{1 "Explicit" rules and rule-guided behaviour}

R-behaviour is a process characterised by the following properties: (1) it develops in more than one step; (2) it is explicit; (3) it is (comparatively) slow and effortful. Let us examine them in some detail.

(1)More than one step

Let us use provisionally the term "rule" to indicate any representation of an action type A in a situation type $\mathrm{S}$ which a concrete action has to satisfy in order to be correct; in other words, any model setting the features of correct action. 
The agent consciously experiences R-behaviour as a process developing in more than one step. We can sketchily summarise and describe these steps as rule formulation, reasoning, decision and observance.

Rule formulation. The agent comes to the mental representation of an action type A in a situation type $\mathrm{S}$, satisfied by the circumstances she actually faces, conceiving it as the rule to be followed, the model setting the features of the correct action to be done in the actual situation. In other words, the agent comes to believe that the right thing to do presently is to follow the rule which prescribes the doing of $A$ in $S$ (i.e., to act in a way that satisfies the represented action type). ${ }^{6}$ The rule is, moreover, somehow linguistically structured: the agent has in mind (already formed or immediately available) an expression of both $\mathrm{A}$ and $\mathrm{S}$ in natural language terms ("Stop at a red light", "Add two to the last number of the sequence"), or in another shared symbolic code. In short, the agent comes to the (mental) formulation of the rule to be followed. Reasoning. The agent uses the rule to discriminate between two alternative courses of action, both believed to be possible: to follow or not to follow the rule (i.e., to act or not to act in a way that satisfies the represented action type). In order to make a choice, she compares then the options, weighing the pros and cons: a more or less articulated "(practical) reasoning" in a very wide sense of the term (it may be nothing more than a flash on a few imagined scenarios, or an accurate selection and balance of the most plausible ones; it could confirm the validity of the rule, or lead her to begin a new process of reasoning in search of another rule, etc.). Decision and observance. The agent finally comes to the decision to follow the rule and effectively follows it (i.e., she decides to perform presently a certain action because it satisfies the action type A that should be performed in the situation type S, satisfied by the circumstances she actually faces, and her decision effectively leads her to perform it). ${ }^{7}$

Given its structure, R-behaviour can also be said to be a threefold "explicit" process guided by a threefold "explicit" standard of correctness.

The rule guiding R-behaviour can, in fact, be said to be "explicit" in three different senses: because it is conscious, because it is (mentally) formulated, and because it functions as a reason.

(a) Explicit as "conscious". During the process, the agent has conscious access to the rule. This is not to be meant only in the sense that she somehow consciously feels that she is acting appropriately (as we shall soon see, such a feeling could also be present in N-behaviour). What is peculiar to R-behaviour is, rather, that the agent has, during the process, a conscious mental representation of the features of a possible action in a possible situation (i.e., a conscious mental representation of an action type $\mathrm{A}$ in a situation type $\mathrm{S}$ ), which a concrete action has to satisfy in order to be correct, and she consciously fits her actual behaviour with that representation. She consciously "looks with the mind's eye", so to speak, at the model for her action, and consciously conforms to it.

(b) Explicit as "(mentally) formulated". The agent has in mind, already formed or immediately available, an expression of both $\mathrm{A}$ and $\mathrm{S}$ in natural language terms, or in another shared symbolic code. She is, therefore, ready to communicate in an abstract, descriptive way the features that make her action correct.

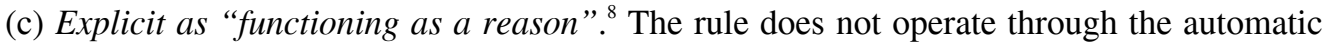
production of the prescribed action. It is, instead, the starting point of a conscious mental comparison between different options (i.e., a more or less articulated reasoning), which could, in principle, lead to the decision both to follow and not to follow the rule. Using Searle's words, " there is a gap in the process: the agent stops her acting and "looks" at the rule, holds it for a while in theoretical space, imaging and balancing the courses of action discriminated by it, taking eventually into account reasons for and against, sometimes starting a new process which can lead to the identification of another rule, etc. In short, the rule functions properly as a reason for action. ${ }^{10}$

24 Being guided by a threefold explicit rule, R-behaviour can correspondingly also be said to be an "explicit" process in three different senses: transparent, discursive, ratiocinative. It is "transparent" because it is guided by a consciously accessed ("visible") rule. It is "discursive" 
because it is guided by a rule which is already formulated in an "inner discourse" and ready to be formulated in a public discourse. It is "ratiocinative" because it involves reasoning, in which the rule functions properly as a reason.

(3)Slow and effortful

R-behaviour is also consciously perceived as a (comparatively) slow and effortful process: the steps through which it develops take some time and the reasoning requires some mental effort, more time and more effort than what is needed for an automatic reaction; moreover, the conscious decision to follow the rule can be costly and even painful. Such an introspective appearance of slowness and effort is strictly correlated with behavioural features, external signs perceptible by an observer: the action is not fluent, there is a perceptible gap, a kind of hesitation preceding acting, and sometimes unskilful execution; prior to and possibly during the action, the agent shows signs of mental concentration (and sometimes of stress or even pain).

\subsection{Norms and norm-conforming behaviour}

R-behaviour develops in more than one step, is threefold explicit (transparent, discursive, ratiocinative), is slow and effortful. By contrast, $\mathrm{N}$-behaviour (1) develops in one single step, (2) is threefold tacit (opaque, dumb, automatic), (3) is fast and effortless.

(1) One step

The agent consciously experiences $\mathrm{N}$-behaviour as developing in one single step, the automatic, spontaneous performance of an action: facing circumstances satisfying a situation type $\mathrm{S}$, the agent immediately, without any conscious deliberation and decision, acts in a way that satisfies an action type A. The action, however, is not accidental, but responds to a general disposition: facing $\mathrm{S}$, the agent regularly does or has the impulse to do A. In short, the agent tends to spontaneously replicate the scheme "A in S".

But this is not enough. The disposition underlying cases of N-behaviour is, indeed, something more than a factual regularity: it has, differently from other kinds of automatic actions, a normative character. Firstly, the agent, being able to discern those concrete behaviours which, in S, satisfy A and those which deviate from A, has the disposition to feel, or judge intuitively, that the former are correct (appropriate, right) and the latter incorrect (inappropriate, wrong). Secondly, these very normative feelings or judgments contribute to the replication of "A in S": if the agent, facing $\mathrm{S}$, deviated from $\mathrm{A}$, she would have a feeling of wrongness which would cause her to adjust her behaviour until it satisfies A; if, instead, she conformed to A, the action would proceed fluently, eventually accompanied by a feeling of appropriateness. In short, the agent tends to act spontaneously in a way that is (or better yet: that the same agent would consider to be) correct, because this is (it would be considered to be) the correct way of acting. ${ }^{11}$ (2)Tacit

Given its structure, N-behaviour appears to be the outcome of some internal mechanism which controls (i) the spontaneous identification (of tokens) of both A and S, (ii) the spontaneous replication of $\mathrm{A}$ when $\mathrm{S}$ is obtained, and (iii) the intuitive judgment that those concrete behaviours which, in S, satisfy A are correct and those which deviate from A are incorrect, with (iii) being, in some way, a contributory condition of (ii).

We could also describe this mechanism by saying that the agent's behaviour is guided by a mental representation of the scheme "A in S", which is "functionally analogous" to a rule: it functions as a model setting the features of the correct action. But, differently from a rule guiding R-behaviour, such a rule-like scheme is "tacit" (implicit, inexplicit) in three different senses: it is unconscious, it is unformulated, it does not function as a reason. Correspondingly, N-behaviour appears to be a threefold "tacit" process: opaque, dumb, not ratiocinative or automatic. Let us try to clarify the point.

31 (a) Tacit as "unconscious". The agent has no conscious access to the scheme she conforms to. This is not to mean that the action and its normative character are completely unperceived. The action is consciously performed, and the agent could also feel that she acts appropriately; she could even think that her ability to act and her feeling of appropriateness depend on some rule-like scheme deeply encoded in her mind. But, she cannot be said to be looking at it "with 
the mind's eye". She has, at least during the process, no conscious mental representation at all of a possible action $\mathrm{A}$ in a possible situation $\mathrm{S}$ as the action to be performed. Consequently, she does not consciously fit her behaviour with any model: she just directly acts in the correct way. For this very reason, N-behaviour can be said to be an "opaque" process: during the process, the scheme that the agent conforms to remains, as such, "invisible" to her.

(A point should be clarified. She who conforms to a norm can be said to have conscious access to it somehow: she is able to conform to the norm and recognise the correctness of her action. ${ }^{12}$ But she is not able to consciously discern the features that render it correct, keep them in mind and use them as a model. In order to distinguish between these two different forms of access, we can talk of "intellectual" (or "analytical" or "abstract") access for the form in which rules are accessed, and of "non-intellectual" (or "synthetic" or "concrete") access for the form in which norms are accessed.)

(b) Tacit as "(mentally) unformulated". The implemented scheme, by consequence, remains also unformulated: during the process, the agent does not have in mind an expression in natural language terms (or in another shared symbolic code) of both A and S. She is, therefore, not ready, or perhaps not able at all, to communicate in an abstract, descriptive way the features that make her action correct. ${ }^{13}$ She is only ready to indicate by ostension a concrete exemplar of correct behaviour. In this sense, $\mathrm{N}$-behaviour appears to be a "dumb" process.

(c) Tacit as "not functioning as a reason". The scheme is automatically implemented, leaving no room for a previous check on whether the action to be performed is or is not the right one to take, no room for thinking whether to act or not to act conforming to the scheme, weighing the reasons for and against. Facing S, the agent just does A spontaneously, without any reasoning. In this sense, the scheme "A in S" does not function properly as a reason, and N-behaviour appears not to be a ratiocinative, but an automatic process. ${ }^{14}$ It is precisely in virtue of their being "tacit", in the sense above described, that the schemes underlying cases of N-behaviour can also be said to be "embodied": ${ }^{15}$ because they manifest themselves directly through the performance ("embodiment", concrete execution) of correct actions, without the intermediation of conscious mental activities, such as reasoning and decision-making, explicitly representing them as models to be followed. On the other hand, it is precisely in virtue of their being based on "tacit" schemes that cases of N-behaviour can be said to be "normative facts": ${ }^{16}$ because the standard according to which they count as correct is accessed directly in their concrete, factual execution (the measure of their correctness lies in their very factual instantiation).

(3)Fast and effortless

N-behaviour is also perceived as a (comparatively) fast and effortless process: the agent acts immediately without thinking about it, and the action requires less mental effort than the effort needed for reasoning and conscious control. Such introspective appearance is strictly correlated with behavioural features: fluency, lack of hesitation, no sign of mental concentration. ${ }^{17}$

Following Celano, we can use the term "norm" to indicate tacit rule-like schemes underlying cases of N-behaviour (hence its name: norm-conforming behaviour). We can use the term "rule" in two senses: more generally, as a commonsensical term indicating any model setting the features of correct actions; and more specifically, as a technical term indicating only explicit models guiding R-behaviour (hence its name: rule-guided behaviour). I shall occasionally refer to the scheme A in S as the "content" of rules or norms.

\subsection{Dynamics of rules and norms}

This is, however, only a first approximation on the opposition between R- and N-behaviour, rules and norms, as it emerges in Celano's paper. Three further clarifications will deepen our understanding of the notions, making their relation more dynamic than it has so far appeared. (1) R-behaviour and N-behaviour should not be thought of as mutually exclusive mental processes in two senses.

40 First, they can operate together as a network of interconnected processes determining the same action. Let us take a swimming training as an example. The trainer gives the athletes their first 
task of the day: " $2000 \mathrm{~m}$ of front crawl". The athletes understand his utterance as establishing a rule and, having it in mind, after ritually protesting against such a boring task and its ritual, inflexible reiteration, start swimming: a case of R-behaviour. But, in swimming, they do not follow a set of rules specifying which precise sequence of movements instantiates a front crawl stroke; they just replicate it automatically, with the underlying disposition to have a feeling of wrongness in the case of incorrect performance, and possibly a feeling of appropriateness in the case of correct performance: a case of N-behaviour. Furthermore (Celano argues, following Wittgenstein), any instance of R-behaviour is necessarily interconnected with, must rely upon, some form of N-behaviour: to avoid infinite regress, there must necessarily be, at some point, an understanding of the concepts appearing in a rule, which does not consist in following other rules specifying their meaning, but which consists in an automatic (and shared) discrimination between cases which do constitute a token (i.e., a correct application) of the concept and cases which do not. In short, all rules necessarily rely on a background of norms. Let us call this "tacit normative background".

Second, one and the same scheme "A in S" could function for one and the same agent at different moments either as a rule or as a norm. Think, for example, about a Lewis convention: the relevant behaviour, Celano says, can initially be performed under the guidance of proper reasoning (R-behaviour) and then become automatic (N-behaviour) ${ }^{18}$ but, the agent could, at some moment, be able to stop the habit and make again explicit the rule she follows (again R-behaviour). In other words, one and the same scheme "A in S" for one and the same agent can be subject to a "functional shift" from rule to norm and vice versa. A behavioural pattern, initially performed under the explicit guidance of a rule, and reiterated over and over, becomes tacit with the passing of time without losing its normative character: a rule is turned into a norm (this is exactly what acquiring an ability amounts to). The agent, having internalised $\mathrm{A}$ in S as a norm, suspends its automatic application, makes its content explicit trough introspection, deliberates about it and finally decides to conform: a norm is turned into a rule (this is exactly what taking a critical stance on our own habits and prejudices amounts to). But, we should be aware of a crucial point. There will be cases in which the functional shift from norm to rule and from rule to norm can occur freely: some of our norms can be made explicit, and some of our rules can become tacit. We can, however, imagine that in other cases, given the structural or contingent limitations of our mind, the shift cannot take place. Some rules cannot be turned into norms: we cannot automatise some behavioural patterns that we otherwise recognise and follow as explicit rules (because they are "too complicated", because they are "too counterintuitive", and so on). And some norms cannot be turned into rules: we cannot make explicit some of the norms we conform to (i.e., we cannot consciously access and formulate their content, and sometimes we cannot even suspend their automatic application). Let us call them "the deep normative background": ${ }^{19}$ the part of our tacit normative background which we can neither make explicit nor describe nor consciously access (in the "intellectual" form), but which we can only directly apply and recognise in its correct applications (i.e., we can only access it in the "non-intellectual" form).

(2) So far, we have drawn a sharp distinction between R- and N-behaviour on the basis of a set of opposed properties: more than one step/one step, explicit/tacit (transparent/opaque, discursive/dumb, ratiocinative/automatic), slow/fast, effortful/effortless. However, this sharp distinction between R- and N-behaviour does not exhaust the possibility of the same properties being combined. In fact, we can easily imagine cases of normative behaviour which share the properties of both $\mathrm{R}$ and $\mathrm{N}$-behaviour.

Let us consider, for instance, the following pattern. The agent does A in S; in her doing A in $\mathrm{S}$, she does not consciously fit her action with a consciously accessed model; nevertheless, her behaviour is normative (she has the disposition to regard as correct those actions which, in S, satisfy A and as incorrect those which do not, and this disposition contributes causally to her doing $\mathrm{A}$ in $\mathrm{S}$ ): these are properties of N-behaviour. Her action, however, is neither fast nor effortless: facing $\mathrm{S}$, she hesitates before doing A, showing signs of mental effort, which are properties of R-behaviour. Let us think about a different pattern. The agent does $\mathrm{A}$ in $\mathrm{S}$ spontaneously, having no doubt about what to do, and acts fast and effortlessly. This seems to 
be a clear case of N-behaviour. She, however, consciously accesses the rule which prescribes the doing of A in S, and observes it intentionally, which are properties of R-behaviour. It is not difficult to imagine more examples of "hybrid" combinations. ${ }^{20}$

Given this varied landscape, the sharply differentiated R- and N-behaviour can be better conceived as paradigms: they show, in the clearest way, the sense in which a normative behaviour and the underlying standard of correctness can be said to be "explicit" or "tacit", and offer an approximate grid for distinguishing and classifying non-paradigmatic cases in virtue of their reputed similarity with either of the two paradigms.

Let us return to our first example. Let us suppose that, in some cases, if asked, the agent can immediately make explicit her mental processes: she can consciously access the scheme she has conformed to, she can even express it in an abstract way, reaffirm her decision to conform and possibly recall the reasons supporting her decision (deeply engaged in a discussion, I stop at a red light; no one is coming; I go a step further, then I stop; although I have paid no attention to my reasoning, I can immediately say that I was inclining to violate the rule, but then I decided to conform). This type of case appear to be very close to paradigmatic Rbehaviour. It shares, first, the same behavioural features of slowness and effort; second, the agent is immediately ready to make explicit the scheme she has conformed to, although, during her acting, she had no conscious access to it. The agent, we could suppose, followed a rule which, albeit not consciously accessed, was just on the "fringe of consciousness": it seems appropriate to talk of "opaque" R-behaviour. (The example is not arbitrary. In fact, Celano seems to admit the possibility of a behaviour being guided by a rule, which is not, however, consciously accessed: a case of "opaque" R-behaviour?). ${ }^{21}$

Let us suppose that, in other cases, the agent cannot at all, or cannot easily, make the scheme explicit. Let us imagine, for instance, a swimmer who knew how to swim a perfect front crawl stroke. After a few month long break, she takes up swimming again and tries to repeat the perfect stroke, but fails. She continues trying over and over until she succeeds. During her trying, she had no explicit rule in mind and was guided only by her feelings of appropriateness or wrongness. After she succeeds, she is not able at all to describe the perfect stroke in an abstract way, she is only able to perform it again and state with (for a non-expert athlete, astonishing) certainty that it is the perfect stroke. This case is far from paradigmatic Rbehaviour. The rule was neither explicit nor on the fringe of consciousness, and it, therefore, seems more appropriate to talk about a norm, a norm still stored in the mind and susceptible to recalling, but provisionally unavailable (i.e., inaccessible even in the "non-intellectual" form). This is a case of "slow \& effortful" N-behaviour.

But, let us imagine now a performing artist in search of a new figure. After having tried different and unsatisfactory ones, she finally finds the "right" solution. During her search, she had no explicit rule in mind and was guided only by her feelings of appropriateness or wrongness. After she finds the right solution, she is neither able at all to describe it in an abstract way nor to explain why it is the right one, she is only able to perform it again and repeat that it is the right figure. It seems odd to say that, in so doing, she has followed a norm which set that precise solution as the right one. The solution is genuinely new and is probably not the only one that would have been approved. It seems more appropriate to say that the artist has tried to fit the performed figure with a set of indeterminate normative standards, which appear to be very similar to norms, because they are neither formulated nor otherwise consciously accessed, and because they control intuitive judgments of correctness. This is another yet different case of "slow \& effortful" N-behaviour. (This example is not arbitrary either. In proposing "acting with style" as an example of N-behaviour, ${ }^{22}$ Celano seems to admit implicitly the possibility that N-behaviour might be effortful. Although acting with style does not amount to following a set of explicit rules, it is not always a matter of finding immediately the right solution either: sometimes it requires a series of tries, which are not guided by explicit rules, but by sound intuitions.)

However, other cases are more difficult to relate either to paradigmatic R-behaviour or to paradigmatic N-behaviour: they seem to fall within the domain of indistinction between the two. Let us return to the odd case introduced above. The agent acts fast and effortlessly, 
but she follows intentionally a consciously accessed rule or at least she can make the rule immediately explicit, expressing it and reaffirming her decision to conform. The teacher, for instance, orders the student to stand up and she spontaneously conforms. If asked, however, the student immediately explains that she stood up because the teacher ordered her to stand up and she ought to obey such an order. It seems natural to think that she was following a rule which is either consciously accessed or "just on the fringe of consciousness". Should we interpret the case as "fast \& effortless" R-behaviour? At the same time, however, it seems appropriate to say that the student has conformed to a norm (she has the "habit of obeying" teacher's orders). Should we rather say that it is a case of "transparent" N-behaviour?

(3) This way of framing the opposition between R- and N-behaviour, rules and norms regarding them as paradigms setting a grid for intuitively classifying different types of non-paradigmatic cases of normative behaviour in a range of similarity - can appear quite unsatisfactory. A better account should, to some degree, make explicit the criteria of similarity, the core features, if any, of R- and N-behaviour, in virtue of which non-paradigmatic cases should be ascribed to either of the two concepts. Such criteria should be capable of clearly framing at least the most important, if not all, non-paradigmatic cases which, like our last example, seem to fall within the domain of indistinction. Well, I think that such criteria are latent in the paper and can be drawn with little effort.

In describing norms, Celano refers fleetingly to the distinction - made famous by D. Kahneman's recent book Thinking, Fast and Slow - between two different "systems" in the mind, which operate by producing mental processes with different introspective and behavioural features: System 1 produces automatic, quick and effortless mental processes, with "no sense of voluntary control", and System 2 produces slow and effortful mental processes, involving "the subjective experience of agency, choice and concentration". ${ }^{23}$ Kahneman's model, which has clearly influenced Celano's notions, also suggests a particularly appropriate way of improving the opposition between R- and N-behaviour, rules and norms, so as to better frame their interaction and the "hybrid" cases exposed above.

Let us assume that paradigmatic $\mathrm{R}$ - and $\mathrm{N}$-behaviours are realised (produced, implemented) by different types of physical (neural) structures, regardless of what their differences may be (different types of neural circuits, different types of computational patterns, etc.). Let us call them R-structure and N-structure respectively. R-structure is something akin to Kahneman's System 2: it operates slowly and effortfully in producing normative behaviours which are explicit or which are such that they become explicit if they become the focus of conscious attention (they can also develop on a preconscious level, "just on the fringe of consciousness"). $\mathrm{N}$-structure is something akin to Kahneman's System 1: it operates fast and effortlessly in producing tacit normative behaviours, and is, as such, incapable of making these behaviours explicit. These are essential features of R-and $\mathrm{N}$-structures, the way in which they necessarily function in virtue of their physical constitution.

Let us try to use this sketchy framework for explaining the dynamics of rules and norms that we have encountered above. The functional shift from norm to rule can be explained as the construction, on a previously existing $\mathrm{N}$-structure, of an $\mathrm{R}$-structure of the same content which can inhibit and overrule it. The functional shift from rule to norm can be explained as the construction, on the basis of a previous R-structure, of an N-structure of the same content which can gradually interpose, substitute or even borrow it. The phenomenon of "the deep normative background" can be explained as the physical impossibility of R-structures with certain contents (certain contents - so-called "sub-doxastic representations" - cannot, as such, become explicit). "Opaque" R-behaviour can be explained as an R-structure functioning on a preconscious level ("just on the fringe of consciousness"). The first case of "slow \& effortless" $\mathrm{N}$-behaviour can be explained as a conscious attempt to re-activate a latent $\mathrm{N}$-structure, while the second as a conscious search for a solution, which fits satisfactorily with an underlying set of indeterminate $\mathrm{N}$-structures. The last and most problematical example ("fast \& effortless" R-behaviour or "transparent" N-behaviour?) can be explained by assuming the coexistence of interconnected N- and R-structures of the same content: the first produces the behaviour 
automatically and the second permits the agent to make the behaviour immediately explicit. And so on.

This account of the opposition between R- and N-behaviour allows us to interpret with ease further connotations of rules and norms given by Celano in his paper. Rules, he says, are "intentional" (and "propositional"), but can nevertheless be unconscious (and, I assume, can function unconsciously), while norms are "non-intentional" (and "non-propositional"). ${ }^{24}$ Taking the "intentionality" of rules for granted, Celano does not explain how it should be understood. He clearly does not refer to a wide sense of "intentionality", taken as the capacity of the mind-brain to represent the world (in this wide sense, a norm could also be said to be "intentional"). More specifically, "intentionality" is taken plausibly as the capability of the mind-brain to represent the world explicitly. An explicit rule is "intentional" in this sense because it involves an explicit (consciously accessed, mentally formulated, functioning as a reason) mental representation of a state of affairs $\mathrm{A}$ in $\mathrm{S}$ (the content of the rule). But, Celano admits the possibility of unconscious rules. In which sense can unconscious rules be said to be intentional as well? Because, in virtue of the R-structure which realises them, they can be made explicit: R-structures are capable of producing explicit representations, and unconscious rules are also realised by R-structures. Conversely, a norm can be said to be "non-intentional" because, in virtue of the $\mathrm{N}$-structure which realises it, it cannot be made explicit: $\mathrm{N}$-structures are not capable of producing explicit representations. ${ }^{25}$ In the same way, we can also interpret Celano's further statement that a rule is "propositional", while a norm is "non-propositional" if we take "propositionality" as a kind of intentionality: intentional content is "propositional" if it can be expressed in a that-clause. (In § 3.4, I shall return to the notion of "propositionality" in the context of Celano's paper.)

This, I hope, is a faithful reconstruction of the complex framework emerging from Celano's Pre-conventions. In sum, it includes: (1) a list of interrelated properties (explicit, slow \& effortful vs. tacit, fast \& effortless) setting a general grid for distinguishing, representing and classifying not only "paradigmatic" R- and N-behaviours, but also "hybrid", slightly more differentiated kinds of normative behaviour; (2) a sketchy outline of rule-following as a dynamic and hierarchically ordered network of automatic N-behaviours, eventually sustaining emergent, more or less ratiocinative episodes of R-behaviour; (3) an explorative hypothesis about the underlying mental architecture, conceived as a dynamic and hierarchically ordered network of $\mathrm{N}$ - and R-structures (taken as different types of neural structures, regardless of what their differences may be). Overall, it is a general account of rule-following based on the opposition between rules and norms, R-and N-behaviour. I shall shortly refer to it as the "Rules vs. Norms Framework".

\section{Some comments about the Rules vs. Norms Framework}

In the previous section, I have proposed a systematic reconstruction of the Rules $v s$. Norms Framework. In this section, I shall further argue that the Framework is the core of Celano's Pre-conventions: it is the keystone of his argument; it is, in its own right, one of the paper's main achievements; it marks a decisive step in Celano's work, pointing at new and promising developments.

\subsection{Rules and norms in Pre-conventions}

First, the Rules vs. Norms Framework plays a crucial role in Celano's argument.

The very notion of a pre-convention and its originality depend on the opposition between rules and norms, R- and N-behaviour. On the one hand, pre-conventions are actually nothing but a network of N-behaviours shared by (most) members of a group G, the Gs, and characterised by the "dependence condition": "A in S" is (functions as) a norm for (most) Gs, and what causes "A in S" to become a "norm" for a $\mathrm{G}$ is the fact that "A in S" is already a norm for (most) Gs. The concept of pre-convention depends, therefore, on the concept of N-behaviour, which is defined by the differences between it and the more familiar R-behaviour. (Pre-conventions are just a peculiar network of N-behaviours. We can indeed imagine kinds of N-behaviour with different origins and structures: for example, "natural" N-behaviours, norms we have 
an innate inclination to conform to, or "idiosyncratic" N-behaviours, norms acquired through experience, but not generally shared by the groups that we are members of.) On the other hand, the reason for the originality of Celano's pre-conventions is precisely the account he gives of their structure. Celano's pre-conventions and Marmor's "deep conventions" ${ }^{\prime 26}$ refer basically to the same phenomena. What Celano adds is a different way of explaining them. They cannot be represented, unless metaphorically, as a network of processes, not even unconscious processes, consisting in the application of shared rules or systems of rules (what I have called "Rbehaviour"). They must be represented as a network of processes of a very different kind, not ratiocinative but automatic, and nevertheless normative (what I have called "N-behaviour"). But the opposition between R- and N-behaviour is fully intelligible precisely in the light of, and it gains its theoretical strength in virtue of, the entire implicit account it relies upon: the Rules vs. Norms Framework.

\subsection{Rules and norms beyond Pre-conventions}

The importance of the Rules $v s$. Norms Framework, it seems to me, goes beyond the scope of Pre-conventions. As I hope to have shown, it traces a comprehensive account of rule-following which - although it cannot as yet be considered a complete theory, but rather an outline needing to be improved, deepened, detailed - is, at this stage, coherent, well-structured, intuitively sound. Moreover, its whole design - although arrived at by developing and linking together well-known ideas - seems to me far from trivial.

Let me briefly dwell on this last point. In drawing his concepts, Celano follows two main patterns. The starting point is the idea - based on Wittgenstein's remarks on rule-following interpreted in terms of Searle's "Background abilities" - that our normative practices cannot be exhaustively represented, unless metaphorically, as the acceptance and observance of systems of rules. A relevant part of our normative practices is controlled by "norms", i.e., schemes which are relevantly different from rules because they are tacit (not explicitly represented), but which are also "functionally equivalent" ${ }^{27}$ to rules because they operate as standards of correctness. Such schemes, given their very nature, are opaque and remain in the background. At the same time, they make up the ground on which our rules necessarily rest: a tacit yet necessarily presupposed background underlying our explicit normative practices. Then, in order to shed more light on the nature of norms, Celano resorts to the distinction, made famous by D. Kahneman, between slow and fast mental processes: ${ }^{28}$ while rules are taken as explicit mental representations underlying slow, ratiocinative, transparent rule-following (what I have called "R-behaviour"), norms are taken as tacit schemes underlying a kind of fast, automatic, blind rule-following (what I have called "N-behaviour"). Thus, rules and norms, and with them the tacit background, are openly framed in psychological terms, as different mental structures involved in different mental processes, defined and distinguished on the basis of both their introspective and behavioural properties. So here it is, in its core, the Rules vs. Norms Framework: a psychological account of rule-following focused on the distinction between slow, ratiocinative, transparent R-behaviour and fast, automatic, blind N-behaviour. The idea that our normative practices rely necessarily on a tacit background appears often in Celano's previous works. ${ }^{29}$ But the psychological account provided by the Rules vs. Norms Framework is a plain novelty, and marks a proper turn in his way of framing problems: psychology has taken centre stage, as far as I know, for the first time. ${ }^{30}$

In spite of the growing fortunes of naturalisation programmes, the use of psychological frameworks in accounting for rules and rule-following dynamics is surely not - at least not yet - mainstream amongst scholars in legal philosophy. But, to my knowledge, some aspects of Celano's Framework - especially its focus on the normativity of fully automatic behaviours and the above outline of the mental processes leading to their execution - can be commonly found neither in the legal philosophical debate, nor in that part of psychological literature on rule-following which is easily accessible to non-specialists. ${ }^{31}$

In summary, the Rules $v s$. Norms Framework can be considered, in its own right, a valuable and significant achievement of Celano's Pre-conventions, for it provides a sketchy yet coherent, well-structured, plausible and, in some respects, unusual account of rule-following. Being 
openly framed in psychological terms, it also represents a turn in Celano's work. This turn, I believe, deserves to be deepened by improving the Framework through the adoption of more, and more fine-grained, psychological concepts and models. ${ }^{32}$

\subsection{Further developments}

Finally and most importantly, the Rules $v s$. Norms Framework also traces a very promising path to the further development of some of Celano's old theses and other traditional issues in legal and moral philosophy.

These are two of Celano's main theses which could be usefully reframed by taking into account the Rules vs. Norms Framework:

(1) Celano's version of natural law theory, "transcendental" natural law, ${ }^{33}$ roughly taken as a set of very undetermined (and conflicting) principles presupposed by our normative practices: the "minimal content" of natural law. The tacit normative background is made of contingent norms, which rest upon, Celano says, "natural" frameworks. Such natural normative frameworks are a plausible interpretation of the principles which define Celano's "minimal content" of natural law. It would be interesting (a) to improve the Rules $v s$. Norms Framework by refining and supporting the idea of a natural normative background and by speculating about its possible contents on the basis of the many available theories of moral innatism, ${ }^{34}$ and (b) to use this refined Framework to account for the constraints on moral and legal reasoning supposedly exercised by the natural normative background.

(2) Celano's version of particularism. ${ }^{35}$ The reasonable (correct) application of a rule, Celano argues, necessarily presupposes a distinction between "normal cases", which the rule applies to, and "exceptional cases", which the rule does not apply to. And this distinction cannot be thought of as defined by other rules: it is a matter of norms. It would be interesting (a) to improve the Rules $v s$. Norms Framework with a detailed psychological account of how norms and rules interact in defeasible reasoning, and (b) to develop Celano's particularism along the lines of this refined Framework.

Let us also consider some classic topics in legal theory, such as the debate about the concept of law. The network of normative practices of which law consists cannot fully be represented, unless metaphorically, as the acceptance and reasoned construction of systems of explicit rules. There is an underlying level of tacit normative concerns (Schmitt's "konkrete Ordnung"), and a continuous shift of our practices from one level to another, and sometimes a dramatic disconnection between the two (the space between Hart's social rules and social habits is neither empty nor static). The Rules $v s$. Norms Framework offers a promising grid for better representing this complex dynamics, and an even more promising one if enriched with more fine-grained psychological concepts and models. ${ }^{36}$ Let us take a look at the notion of authority. Authority, taken as legitimate power, is often defined as the capacity of positing valid rules, existing in virtue of a structure of reasons. Roughly, (a) $\mathrm{Y}$ recognises the authority of $\mathrm{X}$ if $\mathrm{Y}$ believes that the very fact that $\mathrm{X}$ prescribes the doing of $\mathrm{A}$ in $\mathrm{S}$ is, normally, a sufficient reason for concluding that, in S, A ought to be done (i.e., for concluding that the rule "A ought to be done in S" is valid); and (b) Y recognises the authority of $\mathrm{X}$ on the basis of further reasons (i.e., a "justification" of X's authority). Such kinds of definitions "rationalise" authority, reducing its dynamics to a train of explicit processes and completely missing the tacit ones which are part of the ordinary use of the concept (e.g., automatic obedience, automatic signs of submission, automatic acceptance of someone's authority, and so on) and the interaction between the two (e.g., the mutual influence of the habit of obeying and the explicit belief in a duty to obey; or the role that, in reasoning about the validity of a rule prescribed by an authority, may be played by the unconscious check of the content of the rule on the basis of tacit normative standards). Once again, the Rules $v s$. Norms Framework provides a promising grid for an account of authority as a normative phenomenon capable of adequately modelling such complex dynamics.

I both predict and wish to see Celano pursue his "psychological turn", developing, following the path traced, more fine-grained psychological models of rule-following based on the distinction between rules and norms, and using the same to shed new light on traditional topics in legal and moral philosophy. 


\subsection{A methodological issue}

Important merits of the Rules vs. Norms Framework are its intuitive soundness and its neutrality with regard to their explanation on a neural or computational level. Both merits depend on the specific way in which the opposition between R- and N-behaviour is framed. Let me roughly distinguish between three different kinds of properties that we can refer to in conceptualising a type of behaviour. The first ones concern the way in which a particular behaviour is subjectively experienced by the agent. These I have occasionally called "introspective" properties. The second ones concern a particular behaviour's external signs perceptible by an observer. These I have called "behavioural" properties. But introspective and behavioural properties are usually thought of as corresponding to (depending on, supervening upon) properties of a very different kind: neural or computational features of the physical structures which are supposed to produce the behaviour (e.g., the distribution of the relevant neural networks, activation patterns, digital or analogue information processing, and so on). Let us call them "inner" properties.

The notions of R- and N-behaviour have been defined by referring only to either actual or dispositional introspective (explicit vs. tacit) and behavioural (slow \& effortful vs. fast \& effortless) properties. And very apparent ones: a conscious experience framed in terms very close to a commonsensical psychology that everyone is able to use, reducing unusual concepts requiring special philosophical competence to minimum; and external signs easily perceptible by a common observer without the help of special scientific expertise and technical instruments (such features as blood pressure or pupillary response). Furthermore, no hypothesis has been advanced about the inner properties of the physical structures which produce R- and Nbehaviour. This is not to be taken as excluding introspective and behavioural properties, which characterise R- and N-behaviour, corresponding to (depending on, supervening upon) relevantly different inner properties. We have indeed assumed that they do (§ 2.3). But, we have carefully avoided committing ourselves to a precise theory about what exactly such inner properties are. They have been treated as completely opaque and have, as such, not played a part in discriminating cases of $\mathrm{R}$ - from cases of N-behaviour: a particular behaviour counts as an instance of R- or N-behaviour depending on its (actual or dispositional) introspective and behavioural properties, which are assumed to supervene upon certain inner properties, regardless of what they may be.

From being thus defined, the notions of R- and N-behaviour, rules and norms, gain a strong intuitive soundness. And this is not a minor virtue for notions which, albeit drawn in psychological terms, are meant to be used not by professional psychologists or neuroscientists, but by legal and moral thinkers. Every attempt at refining them should take this into consideration, balancing between the value of a more precise neuropsychological account and the advantage of easy accessibility. ${ }^{37}$

Moreover, the notions of R- and N-behaviour, rules and norms, do not imply any hypothesis about the inner properties of the structures which realise them. They can, in this sense, be said to remain neutral about the matter. This also seems to me to be a virtue: it is not easy, not to say impossible, for a non-trained student to gain mastery over the enormous and rapidly growing tools and materials of the neuro- and cognitive sciences necessary to support in a serious way hypotheses about the inner properties of a particular behavioural process. It is better to remain modestly silent as much as possible.

In this regard, the only criticism I have to level at Celano regards his jargon in general and his relaxed use of the concept of "propositionality" in particular.

According to Celano, as we have already seen, rules are "propositional", while norms are "nonpropositional". A "propositional" rule can, moreover, also be "unconscious" 38 (I assume that the idea of an "unconscious rule" includes the possibility of it producing a behaviour without being consciously accessed). Let us call it the "propositionality thesis" (P-thesis). Celano does not explain how the P-thesis is to be understood, taking it for granted. His only suggestion is that to be "propositional" is to be "expressible in a that-clause". ${ }^{39} \mathrm{I}$ have proposed above, in $\S 2.3$, a possible interpretation of the P-thesis which is fully congruent with the other features attributed to rules and norms: "propositionality" is a kind of "intentionality", and a rule is 
"intentional" in the sense that it is stored in the mind in such a way that it can be made explicit, while a norm is not. The problem is that, given the vagueness of Celano's suggestions and the different available accounts of the concept of "proposition" (one of the most obscure and contested in contemporary philosophy), this interpretation of the P-thesis is far from obvious. Other interpretations come easily to mind. ${ }^{40}$

According to one of them, a standard of correctness counts as "propositional" if it is realised by a string of mental symbols somehow encoded in our brain, which shares the same syntactical, semantic and functional properties of the explicit rule "If S, then A ought to be done". In other words, a standard of correctness counts as "propositional" if it is something akin to an explicit rule in a supposed "language of thought". Let us talk of "L-rules". The interpretation of the P-thesis in terms of L-rules is not odd at all: it is perhaps the most obvious for students more familiar with the language of cognitive sciences than with that of analytical philosophy, and can appear natural in a context so deeply impregnated with psychological approaches as Celano's paper. But the interpretation of the P-thesis in terms of L-rules risks upsetting his framework.

Let us consider two theses about L-rules. (1) Fast and effortless tacit normative behaviours are, at least in some cases, the output of the unconscious, automatic and very quick mental computation of L-rules. (2) Some L-rules, called "sub-doxastic" or "sub-personal", are so structured that they cannot be made explicit. Together, these two theses draw a possible (and far from unusual) explanation of the phenomena that Celano is concerned with: fast, tacit, inaccessible normative behaviour. Let us call it the "L-rules explanation".

Let us suppose that we interpret the P-thesis in terms of L-rules. Given that norms are supposed to be non-propositional, N-behaviour cannot be explained in terms of L-rules. Accepting Celano's framework commits us, therefore, to rejecting the L-rules explanation, at least as far as $\mathrm{N}$-behaviour is concerned: $\mathrm{N}$-behaviour cannot be explained as the outcome of the quick computation of sub-doxastic L-rules; it must be the product of some different structure. In this way, the very notions of N-behaviour and norms turn out to be conceptually linked to a very demanding thesis about the inner properties of the physical structures which realise them. Both intuitiveness and neutrality get lost.

80 The problem is marginal in the paper. Another interpretation of the concept of propositionality is available - one which is fully coherent with the whole framework. It underlies, however, important methodological issues. It is highly desirable that the theory of normativity should embrace more, and more fine-grained, psychological concepts and models in order to bridge the gap between old-fashioned metaethics on the one hand, and contemporary moral and social psychology, or even neuro-ethics on the other. But, first, a tremendous amount of grammatical work is required, a careful reframing of the conceptual vocabulary, while paying careful attention to clearly defining key terms, which, in this paradigm shift, can have relevant yet subtle and unperceived semantic changes. Second, as has above been shown, an effort is required to frame our theses in order to avoid unnecessary implications on levels we are not able to manage.

\section{Conclusions}

In writing Pre-conventions, Celano has tried to show that not all standards of correctness are (function as) explicit rules, and that not all normative behaviours (and especially conventional behaviours) can plausibly be thought of as guided by explicit rules. There are standards of correctness, norms, which exist only in their concrete application, and normative behaviours which are the direct and not reflected execution of the correct action. This aim has been, I maintain, fully achieved. But, as I hope to have shown, Celano has arrived at an additional result, which has probably not been intended. He has implicitly set a general account of rulefollowing based on the distinction between rules and norms. This account, although susceptible to improvement by adopting more fine-grained psychological concepts and models, is on the right track with respect to both its content and its form. I look forward to its future applications. 


\section{Bibliography}

Marco BRIGAGLIA, 2011: Ragioni e vincoli. Il potere dialettico del giurista. Roma: Aracne. —_ 2015: Direzione normativa e teoria della mente. Ragion pratica (2015) 44. 103-130.

Bartosz BROŻEK, 2013: Rule-Following. From Imitation to the Normative Mind. Copernicus Center Press: Krakow.

Bruno CELANO, 2002: 'Defeasibility' e bilanciamento. Sulla possibilità di revisioni stabili. Ragion pratica (2002) 18. 223-239.

— 2005: Giusnaturalismo, giuspositivismo e pluralismo etico. Materiali per una storia della cultura giuridica (2005) XXXV. 161-183.

- , 2010a: Consuetudini, convenzioni. Fatti istituzionali, consuetudini, convenzioni. Roma: Aracne. 15-61. First published in: Analisi e diritto 1997. Ricerche di giurisprudenza analitica. Eds. Paolo Comanducci \& Riccardo Guastini. Torino: Giappichelli.

_ 2012: True Exceptions: Defeasibility and Particularism. Essays on Legal Defeasibility. Eds. Jordi Ferrer Beltrán \& Giovanni Battista Ratti. Oxford: Oxford University Press. URL: http://papers.ssrn.com/ sol3/papers.cfm?abstract_id=1759999.

— 2013 (2002): Come deve essere la disciplina costituzionale dei diritti? I diritti nello stato costituzionale. Bologna: Il Mulino.

- 2014: Consuetudine: un'analisi concettuale. Diritto\&Questioni pubbliche (2014) 14. 597-667.

- 2016: Pre-conventions. A fragment of the Background. Revus - Journal for Constitutional Theory and Philosophy of Law (2016) forthcoming. First published in: Ragion Pratica (2014) 2. 605-632.

Robert C. CUMMINS, 1986: Inexplicit Information. The Representation of Knowledge and Belief. Arizona Colloquium in Cognition. Eds. Myles Brand \& Robert M. Harnish. Tucson: University of Arizona Press. 116-126.

Frans B. M. DE WAAL, 1996: Good Natured. The Origins of Right and Wrong in Humans and Other Animals. Cambridge (Mass.)/London: Harvard University Press.

Jonathan HAIDT \& Frederik BJORKLUND, 2008: Social Intuitionists Answer Six Questions about Moral Psychology. Moral Psychology. Volume 2: The Cognitive Science of Morality: Intuition and Diversity. Ed. Walter Sinnott-Armstrong. Cambridge (Mass.)/London: MIT Press. 181-217.

David KAHNEMAN, 2011: Thinking, Fast and Slow. London: Penguin.

Andrei MARMOR, 2009: Social Conventions. From Language to Law. Princeton/Oxford: Princeton University Press.

John R. SEARLE, 1995: The Construction of Social Reality. Harmondsworth: Penguin.

— 2001: Rationality in Action. Cambridge (Mass.)/London: MIT Press.

_ 2004: Mind. A Brief Introduction. Oxford: Oxford University Press.

\section{Notes}

1 Celano 2016: Par. 15.

2 In the whole paper, Celano shifts continuously, although not arbitrarily, from the term "norm" to the term "rule": the first is used with reference to "tacit", "automatic" rule-following which identifies a preconvention, while the second is used with reference to "explicit" rule-following which does not come into play in the case of pre-conventions. The only exceptions are the Introduction (Celano 2016: Par. 1-3), where the two terms are used as synonyms; Argument (II) (Celano 2016: Par. 66-78) and notes 61 and 66 - which refer to Wittgenstein's remarks on rule-following - where the term "rule" is used, generically, with reference to any kind of rule-following.

3 Celano 2016: Par. 15.

4 Celano 2010a: 183 ss; Celano 2014: 613 ss.

5 The distinction between rules and norms, as meant by Celano, regards, in fact, both kinds of actions. See Celano 2016: Par. 17, 49-64, 68-78.

6 In more technical words: she conceives the rule as a "conclusively valid" reason for action.

7 There is something worth noticing here. R-behaviour presupposes the respective ascription of the action to be performed and the actual situation to the action type $\mathrm{A}$ and the situation type $\mathrm{S}$ represented by the rule. Such subsumptive judgments, however, can be made "tacitly", i.e., without any explicit reference to and reasoning about the application of rules which define what counts as a correct instance of $\mathrm{A}$ and $\mathrm{S}$ : a case of N-behaviour. We shall return to this crucial point later $(\S 2.3)$.

8 Here, I follow, with some adjustments, the sense of "explicit" proposed in Cummins 1986.

9 Searle 2001: 14 f., $61 \mathrm{ff}$. 
10 In the above definition of R-behaviour, the first form of explicitness - the property of being mentally accessed - is implied by the other two but does not imply them: in order to be consciously formulated and consciously used as a reason, the rule must be consciously accessed; but we can otherwise imagine cases in which the rule, even if it is consciously accessed, is not formulated and/or not used as a reason. We can also speculate that the mental formulation of a rule or its use in reasoning could occur in an unconscious form as well. I shall discuss odd cases such as these later on in $§ 2.3$.

11 According to Celano, both N-behaviour and other kinds of behavioural patterns, such as innate or acquired reflexes, instincts and habits, are dispositions to act spontaneously in certain ways given certain conditions; but N-behaviour, unlike these other kinds of automatic actions, is the disposition to act in the right way (Celano 2016: Par. 36). I assume that Celano here refers, more precisely, to the disposition to act in a way that the very agent would intuitively consider to be correct (if, in order to have a case of Nbehaviour, the disposition to act in a way that some observer - or the very agent after ex-post reasoning - would consider to be correct were sufficient, the difference between N-behaviour and other kinds of automatic actions would make little sense: every automatic action would count as N-behaviour if it were rationalised ex post!). In the case of a pre-convention, however, the judgment cannot be considered to be merely subjective, because it is, by definition, shared by other members of the group (for $\mathrm{A}$ in $\mathrm{S}$ to be the content of a pre-convention, A should count amongst members of the group as the right action to be done in $\mathrm{S}$ ).

12 In our discussions, Celano strongly stressed that a norm can also be said to be "accessed", but in a different form from that in which a rule is accessed.

13 Celano 2016: Par. 17.

14 Celano does not define explicitly what a norm being tacit amounts to. He only stresses that it is the same sense in which we can be said to "tacitly" believe a proposition which is not stored in our memory as true, but which we would nevertheless immediately and effortlessly recognise as obviously true, without any conscious reasoning. This sense should be distinguished from that in which we can be said to "tacitly believe" propositions stored in our memory as true when they are not presently thought (Celano 2016: Par. 18-19). It should be noted that, in this second sense, rules can also be said to be "tacitly believed" if they are stored in memory as valid rules and can, as such, be recalled.

15 Celano 2016: Par. 15, 24, 42, 80, 87.

16 Celano 2016: Par. 1, 16, 53, 71.

17 Note that the intuitive judgment of correctness underlying N-behaviour is also a case of N-behaviour: a mental action automatically performed under the guidance or control of the same norm, responding to a general disposition. What I have said above about N-behaviour applies, therefore, to this judgment as well: when judging a concrete instance of $\mathrm{A}$ in $\mathrm{S}$ as correct or incorrect, the agent neither compares it to some consciously accessed model nor reasons about it, but directly (intuitively) recognises it as correct or incorrect; moreover, she is not ready to verbalise the reasons supporting her judgment.

18 Celano 2016: Par. 17.

19 See Searle's “deep unconscious” (Searle 2004: $241 \mathrm{f}$.).

20 Let us consider the following cases. The agent is guided by a conscious and quite precise mental picture of an action (she has, for instance, a detailed picture of how her fingers should move in a correct front crawl stroke), but she is not able to verbalise it. The agent is guided by a mental picture which functions as an exemplar which a concrete action has to be sufficiently similar to in order to be correct, but she is not able to discern which of its features are relevant criteria for "sufficient" similarity. In reproducing a melody, the agent is guided by a very precise auditory image of it which she can mentally represent at will, but she does not know any notation system for communicating it in an abstract way. And so on.

21 Celano 2016: Par. 28.

22 Celano 2016: Par. 33-36.

23 Kahneman 2011: $20 \mathrm{f}$.

24 Celano 2016: Par. 79-88 (rules are "intentional", while norms are "non-intentional"); Celano 2016: Par. 28 (being "propositional" is a distinctive feature of rules).

25 This interpretation of the notion of intentionality is strictly analogous to J. R. Searle's "Connection Principle" (Searle 2004: 243 ff.). According to it, "intentionality" denotes, firstly, the way in which conscious mental states represent the world; "intentional" states are, firstly, therefore, conscious mental states. But, secondly, "intentional" can also be said to be any unconscious mental state produced by a physical structure capable of producing the same state in a conscious form. This reference to Searle is particularly appropriate: in distinguishing between rules and norms as intentional and non-intentional standards of correctness, Celano relies precisely on Searle's "Background abilities" (Celano 2016: Par. 79-88).

26 Marmor 2009: 58-78. 
27 Celano 2016: Par. 79-88, referring to Searle 1995: 125-147.

28 Celano 2016: nt. 10 (quoting Kahneman 2011).

29 See, for instance, Celano 2005 and Celano 2013: esp. 104-111.

30 Such a psychological insight acquires, in Pre-conventions, quasi-phenomenological traits. In order to explain what conforming to a norm amounts to, Celano resorts largely to examples which aim to recall and roughly analyse the subjective experience of "embodied" norms. This line of inquiry - which I have tried to emphasise in discussing cases of "odd" normative behaviour which do not fit with the paradigmatic R- and N-behaviours (see § 2.3) - plays a not so negligible role in Celano's paper: the intuitive soundness of the idea of norms as embodied standards of correctness relies largely on the illuminating examples he provides.

31 A relevant exception that I am aware of is the "dual" theory of rule-following recently proposed by the legal philosopher Bartosz Brożek (see Brożek 2013: 44-52). Moving, like Celano, from Wittgenstein’s insights, Brożek indeed distinguishes between two kinds of rules and rule-following. We have, on the one hand, "rudimentary" rules and rule-following (or "rule-observing practices") and, on the other hand, "abstract" rules and rule-following (or "rule-guided behaviours"). Brożek's rudimentary rules and rulefollowing correspond quite well to Celano's norms and the tacit kind of rule-following I call "normconforming behaviour" (more precisely, they correspond to the socially shared norms underlying preconventions). Rudimentary rules are "independent of language" and "followed unconsciously", and rudimentary rule-following is "a "blind' process, almost a reaction", which nevertheless has a normative dimension. Brożek's abstract rules and rule-following correspond to Celano's rules and the explicit kind of rule-following I call (using the same expression Brożek resorts to) "rule-guided behaviour". Abstract rules are "formulated in language" and "followed consciously", and abstract rule-following involves "considering different courses of action, weighing rules for and against incompatible ways of conduct and, ultimately, [...] reasoned decisions as to which rule should be followed". On the basis of this account, Brożek provides a reconstruction of the interaction between rudimentary and abstract rules, which is very close to the one emerging from Celano's paper. For example, abstract rules depend on "existing systems of rudimentary rules" much like the way in which Celano's rules rely on a background of norms. With the passing of time, they can turn into rudimentary rules exactly the way in which Celano's rules can turn into norms. Finally, Brożek argues that imitation - taken as the automatic, unreasoned identification and replication ("embodiment") of patterns of conduct performed by others - is the fundamental mechanism involved in rudimentary rule-following. I am sure that Celano would find this hypothesis intriguing.

32 For example, it would be interesting to conjugate the notions of R- and N-behaviour with J. Haidt and F. Bjorklund's "social intuitionism" (Haidt \& Bjorklund 2008). Above all, the possibility of an automatic ex post and "ideological" justification should be taken into account. The agent acts in a way which she intuitively considers to be correct, having in mind a rule which justifies her action. But, in reality, she does not follow that rule, but a very different norm which she is completely unaware of, and which she cannot openly recognise. For instance, Mr Smith reports to the ticket inspector in a bus that a passenger, clearly an immigrant, has not validated his ticket, firmly convinced that he has done the right thing by denouncing a cheater. But, his action and his feeling of appropriateness have not been related, as he believes, to the fact that he has denounced a cheater, but to the fact that he has denounced a cheater who is also an immigrant. In fact, he has never had even the slightest impulse to denounce a cheater unless the cheater looked like an immigrant! Mr Smith has automatically produced an "ideological" justification for his action, which covers a xenophobic norm deeply encoded in his mind.

33 Celano 2005.

34 For example, I know (through personal communication) that Celano is very intrigued by and has already explored de Waal's theory of the innate basis of moral attitudes, which humans allegedly share with other animals (see de Waal 1996).

35 See, for instance, Celano 2002 and Celano 2012.

36 For a very sketchy and explorative attempt in this direction, see Brigaglia 2011: esp. $311 \mathrm{ff}$.

37 For this methodological argument, see Brigaglia 2015.

38 Celano 2016: Par. 28.

39 Celano 2016: Par. 28.

40 In a very wide sense, at least the norms which are not part of the deep normative background can be expressed in a that-clause which describes their content. I can, for example, say that a swimmer conforms to a norm, according to which "The hand ought to enter into the water finger-tips first, lengthening forward in front of the same shoulder with the middle finger pointing the way to the far end of the pool" (from: http://www.swimsmooth.com/catch.html). It is, however, clear that this is not what Celano is concerned about. In saying that a rule is "propositional", he clearly does not refer to the mere possibility that an observer describes the content of the rule, but specifically to the way in which the rule is represented by the agent when she is guided by it (swimming automatically, the swimmer is not trying to fit her movements with that description!). 


\section{References}

Electronic reference

Marco Brigaglia, « Rules and norms », Revus [Online], in print I 2016, Online since 23 July 2016, connection on 27 September 2016. URL : http://revus.revues.org/3447 ; DOI : 10.4000/revus.3447

Bibliographical reference

Marco Brigaglia, «Rules and norms », Revus, in print I -1, ___.

\section{Author}

Marco Brigaglia

Researcher in Philosophy of Law, Department of Law, University of Palermo

Marco Brigaglia

Dipartimento di Giurisprudenza

Piazza Bologni, 8

90134 Palermo

Italia

E-mail: marco.brigaglia@unipa.it

\section{Copyright}

All rights reserved

\section{Abstract}

Celano's notion of a "pre-convention" is grounded in the opposition between two allegedly different kinds of normative behaviour: observing a "rule" and conforming to a "norm". This opposition plays a central role in Celano's paper, and marks a crucial point in his intellectual trajectory. Nevertheless, it remains largely implicit. In this paper, I try to make it fully explicit, giving a more precise characterisation of both kinds of normative behaviour. I also focus on the importance of distinguishing between them, express some conjectures (or wishes) regarding Celano's future research, and propose a (marginal) criticism.

\section{Index terms}

Keywords : rule-following, fast and slow mental processes, rules and habits, norms and normality 